\title{
A De Novo Missense Mutation in the Inositol 1,4, 5-Triphosphate Receptor Type 1 Gene Causing Severe Pontine and Cerebellar Hypoplasia: Expanding the Phenotype of ITPR1-Related Spinocerebellar Ataxia's
}

\author{
Tessa van Dijk, ${ }^{1}$ Peter Barth, ${ }^{2}$ Liesbeth Reneman, ${ }^{3}$ Bart Appelhof, ${ }^{1}$ Frank Baas, ${ }^{1 *}$ \\ and Bwee Tien Poll-The ${ }^{2}$
}

${ }^{1}$ Department of Clinical Genetics, Academic Medical Centre, University of Amsterdam, Amsterdam, The Netherlands

${ }^{2}$ Department of Pediatric Neurology, Academic Medical Centre, University of Amsterdam, Amsterdam, The Netherlands

${ }^{3}$ Department of Radiology, Academic Medical Centre, University of Amsterdam, Amsterdam, The Netherlands

Manuscript Received: 18 March 2016; Manuscript Accepted: 22 August 2016

We report a de novo missense mutation $($ c.7649T $>A)$ in the inositol, 1,4,5 triphosphate receptor type 1 (ITPR1) gene in a patient with severe pontocerebellar hypoplasia. The mutation results in an amino acid substitution of a highly conserved isoleucine by asparagine (p. I2550N) in the transmembrane domain. Mutations and deletions of the ITPR1 gene are associated with several types of autosomal dominant spinocerebellar ataxia, varying in age of onset and severity. Patients have signs of cerebellar ataxia and at most, a mild cerebellar atrophy on MRI. In contrast, the patient we report here has profound cerebellar and pontine hypoplasia. Our finding therefore further expands the spectrum of ITPR1-related ataxias. ๑ 2016 Wiley Periodicals, Inc.

Key words: pontocerebellar hypoplasia; ITPRI gene; spinocerebellar ataxia

\section{INTRODUCTION}

Mutations in the ITPR1 gene are associated with different types of autosomal dominant spinocerebellar ataxia (SCA) [Hara et al., 2008; Huang et al., 2012; Sasaki et al., 2015] and Gillespie syndrome [Gerber et al., 2016]. Age of onset and severity are variable and hypoplasia/atrophy of cerebellar vermis and hemispheres is mild, if present at all [Huang et al., 2012; Sasaki et al., 2015]. Here, we describe a patient with very severe hypoplasia of cerebellar hemispheres, vermis, and pons, caused by a de novo missense mutation in the ITPR1 gene identified with whole-exome sequencing (WES). This finding was particularly surprising because this patient was, based upon neuroradiological features, suspected to have a form of pontocerebellar hypoplasia $(\mathrm{PCH})$.

De novo mutations in ITPR1 were already described in patients with sporadic infantile onset SCA, who have at most mild cerebellar atrophy, by Sasaki et al. [2015]. This is the first time an ITPR1

\section{How to Cite this Article:} van Dijk T, Barth P, Reneman L, Appelhof B, Baas F, Poll-The BT. 2017. A de novo missense mutation in the inositol 1,4,5-triphosphate receptor type 1 gene causing severe pontine and cerebellar hypoplasia: Expanding the phenotype of ITPR1-related spinocerebellar ataxia's.

Am J Med Genet Part A 173A:207-212.

mutation is found to cause such profound hypoplasia of cerebellum and pons, in severity compatible with $\mathrm{PCH}$. Our finding shows that the phenotype associated with ITPR 1 mutations is even broader than described so far. We, therefore, suggest that, although mutations are probably rare, testing of ITPR 1 should be considered in patients with more severe forms of hypoplasia of pons and cerebellum.

\section{CLINICAL REPORT}

The patient is a 6-year-old girl of non-consanguineous parents with three children. The parents and two older siblings are without cerebellar symptoms. The patient's mother has a microadenoma of the pituitary gland, resulting in hyperprolactinemia. Patient was

Conflict of interest: None.

${ }^{*}$ Correspondence to:

Frank Baas, Department of Clinical Genetics, Academic Medical Centre, Meibergdreef 9, 1105 AZ Amsterdam, The Netherlands.

E-mail: f.baas@amc.uva.nl

Article first published online in Wiley Online Library

(wileyonlinelibrary.com): 9 November 2016

DOI 10.1002/ajmg.a.37962 
born after induced labor at 41 weeks of gestation. Prenatally, an increased nuchal translucency of $4.8 \mathrm{~mm}$ was seen at 12 gestational weeks, but chorionvillus biopsy revealed a normal 46, XX karyotype. Birth weight was 3,130 g (5th-10th centile) and birth head circumference was normal. Apgar score was 10 at $5 \mathrm{~min}$.

At 6 months of age, poor head control and delay in motor development was observed. When examined in the neuropediatric clinic at 16 months, her head circumference was normal $(47.5 \mathrm{~cm}, 50$ th centile); she demonstrated clear developmental delay, with severe truncal titubation and limb ataxia. She was not able to sit unsupported. Deep tendon reflexes were symmetric and brisk. Besides alternating strabismus, there were no ocular abnormalities. She did make eye contact. Developmental age was estimated between 3 months and 6 months. Brain MRI at 16 months showed severe pontine and cerebellar hypoplasia (not shown). There were no supratentorial abnormalities and no signs of cortical atrophy. Because the MRI was compatible with PCH, mutation analysis of the TSEN54, TSEN2, TSEN34, $R A R S 2$, and VRK1 genes was performed, but no mutations were identified. Metabolic screening of blood, urine, and cerebrospinal fluid was normal (including amino acids, sialic acid, $S$-adenosylmethionine, $S$-adenosylhomocysteine, lactate, pyruvate, 5methyltetrahydrofolate, 5-HIAA, HVA, OMD, and GABA). A follow-up MRI at age 3 years and 8 months showed no signs of progression (Fig. 1A and B), specifically no cortical or caudate nucleus atrophy (Fig. 1C), which would be expected in PCH (see Fig. 1D-F for comparison). The vermis inferior is almost absent and the vermis superior showed hypoplasia with superimposed atrophy (Fig. 1B). Hyperintensities in the medu lla oblongata were bilaterally visible on the T2 weighted transversal images, at the site of the inferior olivary nuclei (Fig. 2). At the age of 4 years, she was able to sit and stand with support and could eat by herself. She made sounds but did not speak. Examination showed hypotonia, very brisk deep tendon reflexes, and persisting truncal titubation and limb ataxia. At the age of 6 years, she could speak single words. Hearing was normal. The developmental progress, the absence of progressive microcephaly, and extrapyramidal movement disorders were distinctly different from PCH, especially type 2 [Namavar et al., 2011a]. Because of the lack of a definitive diagnosis, WES of patient and parents (trio analysis) was performed.

\section{MATERIALS AND METHODS}

Total genomic DNA was extracted from peripheral blood leukocytes according to standard protocols. WES was performed on a Solid 5500xl instrument. Enrichment of the exome was performed using the was Nimblegen SeqCap EZ Library v. 3.0 (Roche, Madison, WI). BWA-MEM algorithm [Li, 2013] was used to align the reads to the human reference

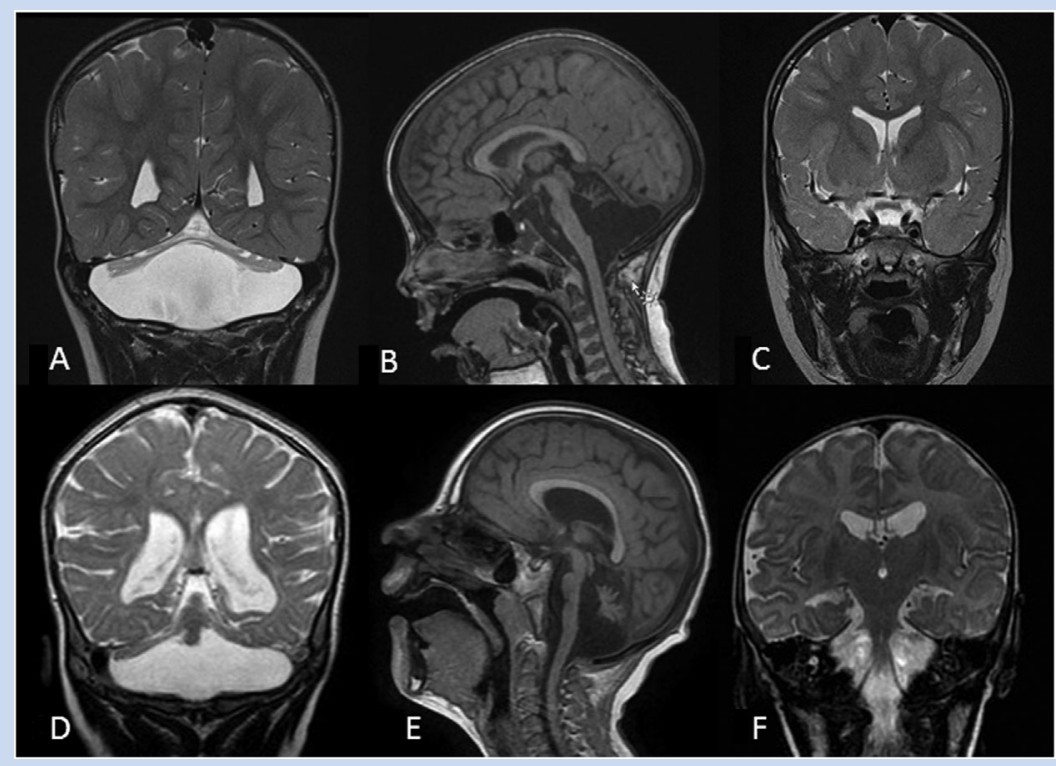

FIG. 1. MRI images of the proband and PCH2 patients. A-C: Proband at age 3 years and 8 months. A: T2 weighted coronal section. Severe hypoplasia of cerebellar hemispheres, without vermal sparing. No signs of cortical atrophy. B: T1 weighted sagittal section. Similar flattening of ventral pons as in PCH2 as well as severe hypoplasia of the cerebellar hemispheres. Vermis inferior is almost absent, vermis superior is hypoplastic with superimposed atrophy. C: T2 weighted coronal section, showing no signs of cortical or caudate nucleus atrophy. D-F: Examples of PCH2 patients. D: T2 weighted coronal section. Severe hypoplasia of cerebellar hemispheres with relative sparing of the vermis [typical 'dragonfly' configuration]. Mild cortical atrophy and enlarged ventricles. E: T1 weighted sagittal section. Flattening of the ventral pons, hypoplasia of the cerebellar hemispheres, and mild atrophy of the cerebellar vermis. F: T2 weighted coronal section. Mild cortical atrophy with atrophy of the head of the caudate nucleus, contributing to the ventricular widening at this level. 


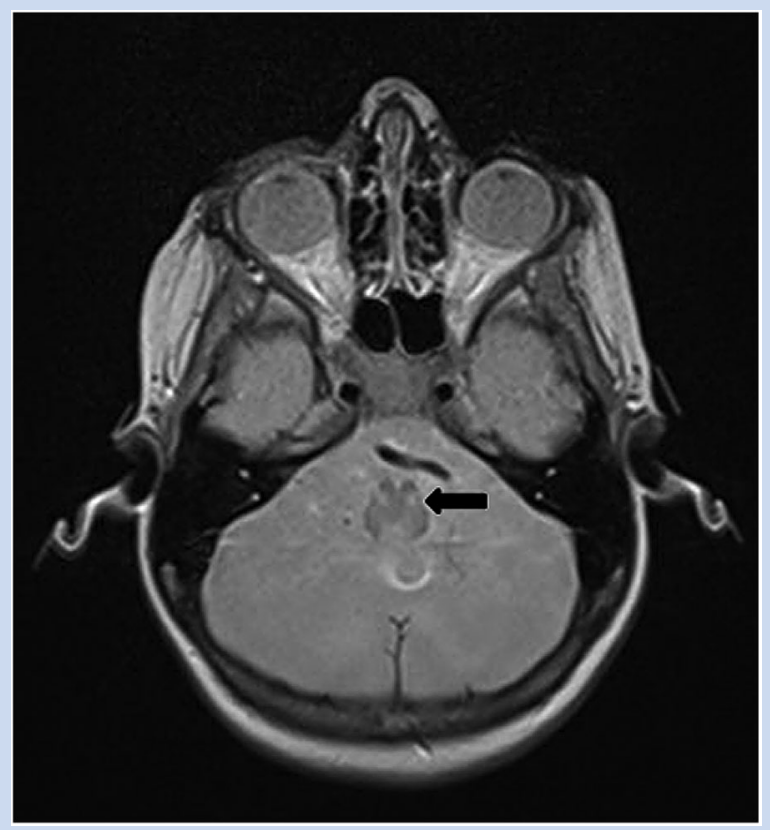

FIG. 2. Bilateral hyperintensities of the medulla oblongata T2 weighted axial image of the proband, at age 3 years and 8 months, showing bilateral hyperintensities of the medulla oblongata at the site of the inferior olivary nuclei, indicating secondary loss of inferior olivary neurons.

genome 19. GATK version 2.7-4 UnifiedGenotyper [DePristo et al., 2011] was used for variant calling. Variants were analyzed with IGV version 2.3.10 and Ingenuity Variant Analysis (Qiagen, Redwood City, CA). Potentially damaging homozygous, compound heterozygous, and de novo variants were filtered out. Filter settings are provided in Supplementary Table SI.

The identified missense mutation in ITPR1 was confirmed using standard Sanger sequencing. PCR amplification using standard conditions with the following M13 tailed primers (Forward: GAGGTCTCACTTGAGCTGTGC, Reverse: AGAGTCAAGACGA AGCAGCA). Codon Code Aligner version 5.0.2 was used to analyze the sequenced samples. Parentage was confirmed with STR analysis (Promega, Madison, WI; PowerPlex 16 system ref DC6531).

\section{RESULTS}

Trio analysis of the WES data revealed a de novo missense mutation in exon 56 of the Inositol 1, 4,5- triphosphate receptor type 1 (ITPR1) gene (c.7649T >A, NM_001099952.2), which results in the amino acid substitution of a highly conserved isoleucine by asparagine ( $\mathrm{p}$. $\mathrm{I} 2550 \mathrm{~N}$ ). The mutation was predicted pathogenic by various in silico prediction programs (i.e., SIFT, Polyphen) and was not present in dbSNP (http://www.ncbi.nlm.nih.gov/SNP/) or the ExAC database (http://exac.broadinstitute.org/). Detailed information on WES data analysis is provided in Supplementary Table SII.

\section{DISCUSSION}

Pontocerebellar hypoplasia represents a clinically and genetically heterogeneous group of disorders comprising 10 subtypes (PCH1-10), all characterized by very early-onset of hypoplasia/ atrophy of cerebellum and pons and severe motor and cognitive impairments [Namavar et al., 2011b]. The most prevalent and best characterized subtype is $\mathrm{PCH} 2$ caused by homozygosity for the p.A307S mutation in the transfer RNA splicing endonuclease subunit 54 gene ( TSEN54). Characteristic neuroradiologic findings are severe hypoplasia of the ventral pons and cerebellum often with relative sparing of the vermis, resulting in a typical "dragonfly" configuration (Fig. 1) [Namavar et al., 2011a]. However, great clinical variability exists within and between the different subtypes. In addition, cerebellar hypoplasia is a relatively common unspecific finding occurring in a very heterogeneous group of disorders [Poretti et al., 2014]. The presence of pontine hypoplasia narrows down the options but making a diagnosis is still challenging. Differential diagnostic considerations are metabolic conditions like congenital disorders of glycosylation (CDG1A), mutations in the CASK-gene, or extreme prematurity [Poretti et al., 2014]. In the case of cortical malformations, mutations in RELN, VLDLR, or FLNA can be considered, as well as mutations in genes involved in the organization of microtubuli (e.g., TUBA1A, TUBB3) [Poretti et al., 2014]. Hypoplasia/atrophy of the cerebellum at young age can also occur in early-onset types of SCA. Brainstem involvement however, is an uncommon finding in SCA, but has been described in rare forms of very severe infantile onset SCA2 and SCA7 [Poretti et al., 2015]. Typically, other clinical findings like retinitis pigmentosa or cone-rod dystrophy are present in these conditions [Singh et al., 2014; Donis et al., 2015].

This is the first time a de novo missense mutation in the ITPR 1 gene is reported in a patient with early-onset ataxia and a neuroradiological presentation, suggesting severe $\mathrm{PCH}$. Mutations and deletions of the ITPR1 gene have been associated with several forms of cerebellar ataxia (Table I) [Van De Leemput et al., 2007; Hara et al., 2008; Iwaki et al., 2008; Huang et al., 2012; Sasaki et al., 2015], but cerebellar hypoplasia was at most mild in those patients. The patient we describe here presented with infantile onset cerebellar symptoms, motor development delay, and intellectual disability. Up to the age of 6 years, clinical course is nonprogressive.

The ITPR1 gene encodes the inositol 1,4,5-triphosphate receptor type 1 . The three subtypes of inositol 1,4,5 triphosphate receptors (IP3R1, IP3R2, and IP3R3) are mediated by inositol triphosphate (IP3) [Krizanova and Ondrias, 2003]. They function as intracellular calcium channels involved in the calcium release from various intracellular stores like the endoplasmic reticulum (ER) and the Golgi apparatus [Berridge, 1993, Lencesova and Krizanova, 2012]. They are involved in the regulation of various vital processes [Berridge, 1993; Lencesova and Krizanova, 2012]. ITPR1 has a particular high expression in the cerebellum [Dent et al., 1996; Krizanova and Ondrias, 2003; Lencesova and Krizanova, 2012; Sugawara et al., 2013] and ITPR1deficient mice were shown to have ataxia and other motor deficits [Matsumoto et al., 1996; Ogura et al., 2001].

Heterozygous (partial) deletions of the ITPR1 gene were first described to cause SCA15 in three families by Van De Leemput et al. 


\section{TABLE I. Overview of Spinocerebellar Ataxia (SCA) Subtypes Associated With ITPR1}

Type SCA

SCA15

Deletion $^{2}$

Mutation

\section{SCA29/infantile onset \\ SCA/ataxic cerebral palsy}

\section{Gillespie syndrome}

$\mathrm{PCH}$ with ataxia
Heterozygous missense mutation in IP3-

binding or -coupling domain

De novo missense mutation in IP3binding domain/ biallelic truncating mutations

De novo missense mutation in transmembrane domain
Symptoms

Adult onset cerebellar ataxia, slowly progressive.

Onset of symptoms in first year of life (but occasionally later], variable ID. Nonprogressive.

Neonatal onset of symptoms, cerebellar ataxia, iris hypoplasia, variable ID. Nonprogressive.

Early onset ataxia, delayed motor and mental development. Nonprogressive.
MRI

Mild cerebellar atrophy, vermis

$>$ hemispheres, no pons atrophy.

Mild atrophy of cerebellar hemispheres, vermis and (rarely) pontine tegmentum.

Mild/moderate cerebellar atrophy. Progressive.

Severe $\mathrm{PCH}$; vermis and hemispheres equally affected.
Number of patients reported

$>25$ [e.g., Van De Leemput et al., 2007; Hara et al., 2008]

27 [Huang et al., 2012; Sasaki et al., 2015; Schnekenberg et al., 2015; Barresi et al., 2016; Shadrina et al., 2016]

18 [Gerber et al., 2016; McEntagart et al., 2016]

1
[2007]. Only one missense mutation, located in the coupling domain of ITPR1, has been reported in SCA15 [Hara et al., 2008]. SCA15 is a slowly progressive form of cerebellar ataxia with most often an onset in adulthood [Van De Leemput et al., 2007].

Missense mutations in ITPR1 were identified in three families with a form of congenital nonprogressive spinocerebellar ataxia
(CNPCA) with autosomal dominant inheritance [Dudding et al., 2004; Huang et al., 2012; Shadrina et al., 2016]. Onset of symptoms may vary but is, in contrast to SCA15, often in the first year of life. Based on the earlier onset, this type of SCA was classified as SCA 29 [Sasaki et al., 2015]. The main clinical symptoms were early motor delay and nonprogressive ataxia, sometimes with mild cognitive impairment or learning problems. Other neurological features as

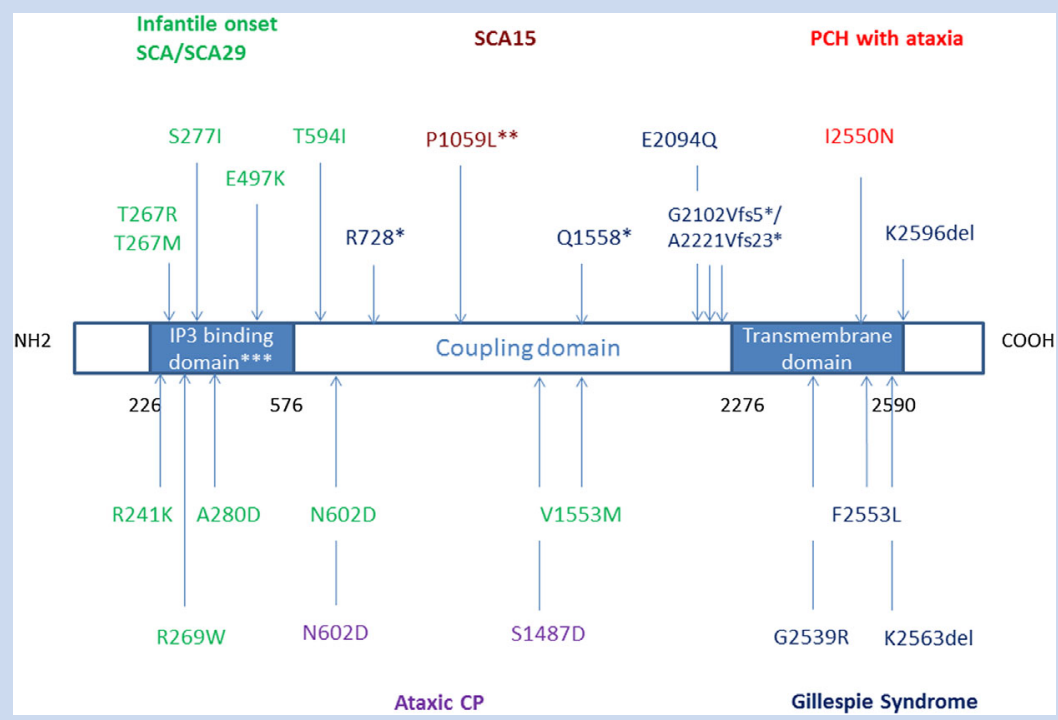

FIG. 3. Overview of amino acid substitutions reported in ITPR1 with associated phenotypes. Showing previously reported amino acid substitutions in the ITPR1 protein found in patients with different types of SCA. CP: Cerebral palsy. [*] Homozygous or compound heterozygous mutations. $\left[{ }^{* *}\right.$ ] Only missense mutation reported in SCA15. Asterisk [ ${ }^{* * *}$ ] IP3 binding domain is roughly indicated and might be interrupted at some points; the exact IP3 region is unknown. 
nystagmus and dysarthria were variably present, while pyramidal tract or sensory symptoms were absent. Brain MRI showed cerebellar vermal hypoplasia in a part of the patient group, which in some patients showed some progression. In all families, missense mutations were localized to the coupling/regulatory domain (p. V1553M and p.N602D, respectively, Fig. 3) [Huang et al., 2012; Shadrina et al., 2016]. De novo missense mutations in the coupling regulatory domain of ITPR1 were also reported in two patients initially diagnosed with ataxic cerebral palsy [Schnekenberg et al., 2015]. Brain imaging including the cerebellum was normal.

In 2015, four sporadic cases of infantile onset spinocerebellar ataxia (IOSCA) caused by de novo missense mutations in ITPR1 were described [Sasaki et al., 2015]. Early symptoms shared by all patients were nystagmus, general hypotonia and tremor/ataxia of head, trunk, and upper extremities. All had a delayed motor and mental development. Brain MRI showed very slowly progressive atrophy of cerebellum, vermis, and pontine tegmentum. The clinical course was nonprogressive, as far as observed. All four identified mutations were located in or near the IP3-binding domain (Fig. 3). Barresi et al. [2016] also recently identified heterozygous missense mutations located in the IP3-binding domain in seven patients from four families. The probands presented with cerebellar symptoms like ataxia and nystagmus in the first year of life. In two families, the mutation was inherited from a parent with later onset of symptoms.

Recently, mutations in the ITPR1 gene were identified in patients with Gillespie syndrome [Gerber et al., 2016; McEntagart et al., 2016]. Gillespie syndrome is characterized by non-progressive cerebellar ataxia, variable intellectual disability, and iris hypoplasia. Brain MRI shows marked cerebellar atrophy (progressive). Both biallelic truncating mutations and heterozygous missense mutations were identified. Almost all missense mutations were located in the distal part of the transmembrane domain, near the mutation in our patient (Fig. 3). The K2563del mutant was shown to affect calcium release when co-expressed with wild-type ITPR1, indicating a dominant negative effect [Gerber et al., 2016]. A dominant negative effect was also suggested for the other missense mutations, based upon in silico predictions considering destabilizing effects and comparison to other known dominant negative mutations in transmembrane channels [McEntagart et al., 2016].

We identified a mutation in the ITPR 1 gene in a patient with a much more severely affected cerebellum and pons than previously found in ITPR1-related ataxias. The variation in the phenotype could be related to the location of the mutations in the protein; however, a clear phenotype-genotype could not be established. One can speculate that missense mutations in the IP3-binding domain and -coupling domain are associated with SCA29/infantile onset SCA, while missense mutations in the transmembrane domain are related to Gillespie syndrome. The mutation in our patient is predicted at the border of the transmembrane ion channel pore domain, in close proximity to the heterozygous mutations found in Gillespie syndrome. Ophthalmologic examination including funduscopy in our patient at the age of 3 years did not reveal any ocular abnormalities (besides mild convergent strabismus).

The c.7649T $>$ A mutation, resulting in the substitution of the highly conserved hydrophobic isoleucine by the polar hydrophilic asparagine, might disrupt the transmembrane domain. This amino acid substitution creates an additional consensus site for $N$-glycosylation (Asn-Asp-Thr) [Gavel and Von Heijne, 1990]. The potential attachment of a hydrophilic oligosaccharide potentially further disrupts organization of the transmembrane domain and interfere with the composition of tetrameric channels.

In conclusion, we broadened the spectrum of ITPR1-related ataxias by identifying a de novo missense mutations in a patient with very severe hypoplasia of cerebellum and pons, mimicking $\mathrm{PCH}$.

\section{ACKNOWLEDGMENTS}

The authors would like to thank the patient and her family for their participation.

\section{REFERENCES}

Barresi S, Niceta M, Alfieri P, Brankovich V, Piccini G, Bruselles A, Barone MR, Cusmai R, Tartaglia M, Bertini E, Zanni G. 2016. Mutations in the IRBIT domain of ITPR1 are a frequent cause of autosomal dominant nonprogressive congenital ataxia. Clin Genet DOI: 10.1111/cge.12783

Berridge MJ. 1993. Inositol trisphosphate and calcium signalling. Nature $361: 315-325$

DePristo MA, Banks E, Poplin RE, Garimella KV, Maguire JR, Hartl C, Philippakis AA, del Angel G, Rivas MA, Hanna M, McKenna A, Fennell TJ, Kernytsky AM, Sivachenko AY, Cibulskis K, Gabriel SB, Altshuler D, Daly MJ. 2011. A framework for variation discovery and genotyping using next generation DNA sequencing data. Nat Genet 43:491-498.

Dent M, Raisman G, Lai F. 1996. Expression of type 1 inositol 1,4, 5-trisphosphate receptor during axogenesis and synaptic contact in the central and peripheral nervous system of developing rat. Development 122:1029-1039.

Donis KC, Mattos EP, Silva AA, Furtado GV, Saraiva-Pereira ML, Jardim LB, Saute JA. 2015. Infantile spinocerebellar ataxia type 7: Case report and a review of the literature. J Neurol Sci 254:118-121.

Dudding TE, Friend K, Schofield PW, Lee S, Wilkinson IA, Richards RI. 2004. Autosomal dominant congenital non-progressive ataxia overlaps with the SCA15 locus. Neurology 63:2288-2292.

Gavel H, Von Heijne G. 1990. Sequence differences between glycosylated and non-glycosylated Asn-X-Thr/Ser acceptor sites: Implications for protein engineering. Protein Eng 3:433-442.

Gerber S, Alzayady KJ, Burglen L, Brémond-Gignac D, Marchesin V, Roche O, Rio M, Funalot B, Calmon R, Durr A, Gil-da-Silva-Lopes VL, Bittar MFR, Orssaud C, Héron B, Ayoub E, Berquin P, Bahi-Buisson N, Bole C, Masson C, Munnich A, Simons M, Delous M, Dollfus H, Boddaert N, Lyonnet S, Kaplan J, Calvas P, Yule DI, Rozet JM, Taie LF. 2016. Recessive and dominant de novo ITPR1 mutations cause Gillespie syndrome. Am J Hum Genet 98:971-980.

Hara K, Shiga A, Nozaki H, Mitsui J, Takahashi Y, Ishiguro H, Yomono H, Kurisaki H, Goto J, Ikeuchi T, Tsuji S, Nishizawa M, Onodera O. 2008. Total deletion and a missense mutation of ITPR1 in Japanese SCA15 families. Neurology 71:547-551.

Huang L, Warman J, Carter MT, Friend KL, Dudding TE, Schwartzentruber J, Zou R, Schofield PW, Douglas S, Bulman DE, Boycott KM. 2012. Missense mutations in ITPR1 cause autosomal dominant congenital nonprogressive spinocerebellar ataxia. Orphanet J Rare Dis 7:67-73. 
Iwaki A, Kawano Y, Miura S, Shibata H, Matsuse D, Li W, Furuya H, Ohyagi Y, Taniwaki T, Kira J, Fukumaki Y. 2008. Heterozygous deletion of ITPR1, but not SUMF1, in spinocerebellar ataxia type 16. J Med Genet 45:32-35.

Krizanova O, Ondrias K. 2003. The inositol 1,4,5-trisphosphate receptortranscriptional regulation and modulation by phosphorylation. Gen Physiol Biophys 22:295-311.

Lencesova L, Krizanova O. 2012. IP3 receptors, stress and apoptosis. Gen Physiol Biophys 31:119-130.

Li H. 2013. Aligning sequence reads, clone sequences and assembly contigs with BWA-MEM2013. arXiv:1303.3997 [q-bio.GN].

Matsumoto M, Nakagawa T, Inoue T, Nagata E, Tanaka K, Takano H, Minowa O, Kuno J, Sakakibara S, Yamada M, Yoneshima H, Miyawaki A, Fukuuchi Y, Furuichi T, Okano H, Mikoshiba K, Noda T. 1996. Ataxia and epileptic seizures in mice lacking type 1 inositol 1,4,5-trisphosphate receptor. Nature 379:168-171.

McEntagart M, Williamson KA, Rainger JK, Wheeler A, Seawright A, De Baere E, Verdin H, Bergendahl LT, Quigley A, Rainger J, Dixit A, Sarkar A, López Laso, Sanchez-Carpintero R, Barrio J, Bitoun P, Prescott T, Riise R, McKee S, Cook J, McKie L, Ceulemans B, Meire F, Temple IK, Prieur F, Williams J, Clouston P, Németh AH, Banka S, Bengani H, Handley M, Freyer E, Ross A, DDD study, van Heyningen V, Marsh JA, Elmslie F, FitzPatrick DR. 2016. A restricted repertoir of de novo mutations in itprl cause Gillespie syndrome with evidence for dominant-negative effect. Am J Hum Genet 98:981-992.

Namavar Y, Barth PG, Kasher PR, van Ruissen F, Brockmann K, Bernert G, Writzl K, Ventura K, Cheng EY, Ferriero DM, Basel-Vanagaite L, Eggens VRC, Krageloh-Mann I, De Meirleir L, King M, Graham JM, von Moers A, Knoers N, Sztriha L, Korinthenberg R, Consortium PCH, Dobyns WB, Baas F, Poll-The BT. 2011a. Clinical, neuroradiological and genetic findings in pontocerebellar hypoplasia. Brain 134:143-156.

Namavar Y, Barth PG, Poll-The BT, Baas F. 2011b. Classification, diagnosis and potential mechanisms in pontocerebellar hypoplasia. Orphanet J Rare Dis 6:50-64.

Ogura H, Matsumoto M, Mikoshiba K. 2001. Motor discoordination in mutant mice heterozygous for the type 1 inositol 1,4,5-trisphosphate receptor. Behav Brain Res 122:215-219.
Poretti A, Boltshauser E, Doherty D. 2014. Cerebellar hypoplasia: Differential diagnosis and diagnostic approach. Am J Med Genet Part C 166C:211-226.

Poretti A, Wolf N, Boltshauser E. 2015. Differential diagnosis of cerebellar atrophy in childhood: An update. Neuropediatrics 46:359-370.

Sasaki M, Ohba C, Iai M, Hirabayashi S, Osaka H, Hiraide T, Saitsu H, Matsumoto N. 2015. Sporadic infantile-onset spinocerebellar ataxia caused by missense mutations of the inositol 1,4,5-triphosphate receptor type 1 gene. J Neurol 262:1278-1284.

Schnekenberg RP, Perkins EM, Miller JW, Davies WIL, D’Adamo MC, Pessia M, Fawcett KA, Sims D, Gillard E, Hudspith K, Skehel P, Williams J, O’Regan M, Jayawant S, Jefferson R, Hughes S, Lustenberger A, Ragoussis J, Jackson M, Tucker SJ, Németh AH. 2015. De novo point mutions in patients diagnosed with ataxic cerebral palsy. Brain 138:1817-1832.

Shadrina MI, Shulskaya MV, Klyushnihov SA, Nikopensius T, Nelis M, Kivistik PA, Komar AA, Limborska SA, Illarioshkin SN, Slominsky PA. 2016. ITPR1 gene p.Val1553Met mutation in Russian family with mild spinocerebellar ataxia. Cerebellum Ataxias 3:2.

Singh A, Faruq M, Mukerji M, Dwivedi MK, Pruthi S, Kapoor S. 2014. Infantile onset spinocerebellar ataxia 2 (SCA2): A clinical report with review of previous cases. J Child Neurol 29:139-144.

Sugawara T, Hisatsune C, Le TD, Hashikawa T, Hirono M, Hattori M, Nagao S, Mikoshiba K. 2013. Type 1 inositol trisphosphate receptor regulates cerebellar circuits by maintaining the spine morphology of Purkinje cells in adult mice. J Neurosci 33:12186-12196.

Van De Leemput J, Chandran J, Knight M, Holtzclaw L, Scholz S, Cookson MR, Houlden H, Gwinn-Hardy K, Fung HC, Lin X, Hernandez D, Simon-Sanchez J, Wood NW, Giunti P, Rafferty I, Hardy J, Storey E, Gardner RJM, Forrest SM, Fisher EMC, Russell JT, Cai H, Singleton AB. 2007. Deletion at ITPR1 underlies ataxia in mice and spinocerebellar ataxia 15 in humans. PLoS Genet 3:1076-1082.

\section{SUPPORTING INFORMATION}

Additional supporting information may be found in the online version of this article at the publisher's web-site. 